\title{
BMJ Open Efficacy of an internet-based self-help intervention to reduce co-occurring alcohol misuse and depression symptoms in adults: study protocol of a three-arm randomised controlled trial
}

\author{
Michael P Schaub, ${ }^{1}$ Matthijs Blankers, ${ }^{2,3}$ Dirk Lehr, ${ }^{4}$ Leif Boss, ${ }^{5}$ Heleen Riper, ${ }^{5}$ \\ Jack Dekker, ${ }^{2,6}$ Anna E Goudriaan, ${ }^{2,3}$ Larissa J Maier, ${ }^{1}$ Severin Haug, ${ }^{1}$ \\ Manuel Amann, ${ }^{1}$ Michelle Dey, ${ }^{1}$ Andreas Wenger, ${ }^{1}$ David D Ebert ${ }^{7}$
}

To cite: Schaub MP, Blankers M, Lehr D, et al. Efficacy of an internet-based self-help intervention to reduce co-occurring alcohol misuse and depression symptoms in adults: study protocol of a three-arm randomised controlled trial. BMJ Open 2016;6:e011457. doi:10.1136/bmjopen-2016011457

- Prepublication history for this paper is available online. To view these files please visit the journal online (http://dx.doi.org/10.1136/ bmjopen-2016-011457).

Received 9 February 2016 Revised 27 April 2016 Accepted 4 May 2016

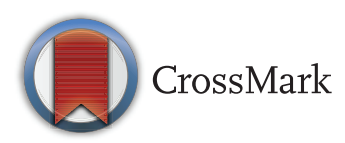

For numbered affiliations see end of article.

\section{Correspondence to} Dr Michael P Schaub; michael.schaub@isgf.uzh.ch

\section{ABSTRACT}

Introduction: In the general population, alcohol use disorder and depression more often occur together than any other combination of a mental illness with a substance use disorder. It is important to have a costeffective intervention that is able to reach at-risk individuals in the early stages of developing alcohol use disorders and depression disorders.

Methods and analysis: This paper presents the protocol for a 3-arm multicentre randomised controlled trial (RCT) to test the efficacy and cost-effectiveness of the combined internet-based self-help intervention Take Care of You (TCOY) to reduce alcohol misuse and depression symptoms in comparison with a waiting list control group and a comparable intervention focusing on problematic alcohol use only. The active interventions consist of modules designed to reduce alcohol use, based on the principles of motivational interviewing and methods of cognitive behavioural therapy, together with additional modules in the combined study arm to reduce symptoms of depression. Data will be collected at baseline, as well as at 3 and 6 months postrandomisation. The primary outcome is the quantity of alcohol used in the past 7 days. A number of secondary outcome measures will be studied. These include the Centre of Epidemiologic Studies of Depression Scale (CES-D) and a combined measure with the criteria of values below the cut-off for severe alcohol use disorder and for CES-D. Data analysis will follow the intention-to-treat principle using (generalised) linear mixed models. In order to investigate the interventions' cost-utility and costeffectiveness, a full economic evaluation will be performed.

Ethics and dissemination: This RCT will be executed in compliance with the Helsinki Declaration and has been approved by 2 local Ethics Committees. Results will be reported at conferences and in peerreviewed publications. Participant-friendly summaries of trial findings will be published on the TCOY websites.

Trial registration number: ISRCTN10323951.

\section{Strengths and limitations of this study}

Take Care of You (TCOY) is a multicentre randomised controlled trial to assess the efficacy and cost-effectiveness of an internet-based self-help intervention that aims to reduce problematic alcohol use and depression symptoms simultaneously.

- A strength of the present study is that the effects of the intervention on alcohol use (disorders) depression, mental health symptoms and intervention satisfaction are assessed in comparison to a waiting list control group, as well as explored relative to a comparable 'alcohol-only' intervention regarding its differential effects.

- A limitation of the study is that only self-report measures are used.

\section{INTRODUCTION}

Mental disorders constitute a major burden of disease with significant effects on physical health and major social and economic consequences. Mental disorders, such as alcohol use disorder and depression, are chronic intermittent conditions that may affect, and are affected by, other chronic diseases, such as cancer and cardiovascular disease. ${ }^{1}$ Taken together, substance use and other mental disorders account for $7.4 \%$ of the total global burden of disease. ${ }^{2}$ Alcohol misuse alone accounts for $5.1 \%$ of the global burden of disease and $5.9 \%$ of all deaths worldwide. ${ }^{3}$ Alcohol misuse in the present protocol is defined as a score of 8 or more on the Alcohol Use Disorder Identification Test $\left(\right.$ AUDIT $\left.^{4}\right)$.

Co-occurring disorders are also called dual disorders, dual diagnosis or comorbid disorders and are diagnosed if at least two 
disorders occur simultaneously in the same person. In the general population, the most frequent of these is the combination of alcohol use disorder and depression and/or anxiety disorder. ${ }^{5-7}$ Comorbidity of alcohol abuse and dependence is two to three times higher for those who suffer from depression than for those in the general population. ${ }^{8}$ Moreover, risky alcohol use is associated with a higher probability of developing affective disorders than for not at-risk users. ${ }^{9}$

In recent years, treatment models that target at-risk populations have been developed and tested in randomised controlled trials (RCTs). ${ }^{10}$ In addition, internetbased interventions for individuals with different forms of problematic substance use $\mathrm{u}^{11-18}$ and/or subclinical mental health symptoms ${ }^{19}$ were recently developed. These internet-based interventions reach at-risk individuals at early stages of potentially more pronounced mental health disorders.

By now, internet-based interventions have been conducted as RCTs to reduce or quit the use of tobacco, ${ }^{20}$ alcohol,${ }^{17}$ cannabis, ${ }^{1315}$ cocaine $^{12}$ and amphetamine-type stimulants. ${ }^{16}$ The greatest effect sizes have consistently been reported for internet-based self-help interventions that aim to control and/or reduce consumption of alcohol misusers by means of cognitive behavioural therapy (CBT) with a pooled effect of Hedges's $g=0.20$ $(95 \%$ CI 0.13 to 0.27$)$, as presented in a meta-analysis. ${ }^{17}$ These interventions have been proven to be safe for adult alcohol misusers. ${ }^{18}$

Computerised and, more recently, internet-based treatments for depression have been developed and proven effective in controlled trials, both for adults ${ }^{21}$ and adolescents. ${ }^{22}$ Richard and Richardson ${ }^{23}$ reported a pooled effect size of $\mathrm{d}=0.56$ (CI 0.71 to -0.41 ) in their meta-analysis of comparisons between internet-based and other computerised psychological treatments versus control groups.

High rates of co-occurring depression symptoms and substance use and associated negative impacts on the course of the illness and treatment outcomes have been well established. ${ }^{24}$ Riper et $a l^{25}$ demonstrated that CBT in combination with motivational interviewing (MI) predominantly delivered in face-to-face therapy-proved effective for treating subclinical and clinical alcohol use disorders and major depressive disorders (combined treatment), as compared with controls, with small overall effect sizes at post-treatment $(\mathrm{g}=0.17$, CI 0.07 to 0.28 for decrease in alcohol consumption and $\mathrm{g}=0.27$, CI 0.13 to 0.41 for decrease in symptoms of depression, respectively). An important finding was that there were analogous effects for subclinical depression and substance use disorders, as this is relevant for the internet-based targeting of such individuals. Furthermore, they found that a greater number of CBT/MI sessions was significantly associated with better alcohol outcomes, but not with the depression outcomes.

For patients with dual diagnosis disorders, integrated treatment-with treatment elements to reduce both alcohol use and depression symptoms-can reduce treatment duration and increase patients' treatment satisfaction to a greater extent than non-integrated treatment. ${ }^{26}$ Thus, integrated treatments may be more cost-effective. A brief internet-based intervention for co-occurring depression and problematic alcohol use in young people $(\mathrm{N}=104)$ had been shown to be effective for both outcomes in the short term when compared with an attention-control condition. ${ }^{27}$ However, no study has been conducted to examine the effects of integrated internetbased self-help interventions in adults. Therefore, our study aims to investigate the efficacy and costeffectiveness of the internet-based self-help intervention Take Care of You (TCOY), which incorporates adherencefocused guidance to target comorbid problematic alcohol use and depression symptoms in adults. The study is designed as a three-arm RCT with a combined intervention for alcohol misuse and depression, an intervention focusing on alcohol only and a waiting list. We expect that the combined intervention will give greater improvements in depression symptoms and treatment satisfaction than the alcohol-only intervention, but with similar reductions in alcohol-related outcomes.

\section{METHODS AND ANALYSES \\ Study design}

A three-arm RCT will be conducted to evaluate the webbased self-help intervention TCOY with and without modules for depression symptoms compared with a waiting list control. Assessments will take place before the allocation to the study conditions (T0), as well as at 6 weeks (T1), and 3 and 6 months (T2/3) after the allocation (figure 1). All procedures involved in the study will be consistent with the generally accepted standards of ethical practice and the trial is registered at Current Controlled Trials and traceable as ISRCTN10323951.

\section{Recruitment of study participants}

Potential participants will be recruited from February 2016 to January 2018 through the corresponding internet health portals or internet websites and by advertisements in commuter newspapers for each country separately. Specific study advertisements will be placed on relevant online health portals in the Netherlands, Germany and Switzerland.

\section{Registration and consent procedure}

Potential participants will first read the study information about the background rationale and then be informed of the following: (1) study inclusion and exclusion criteria (see table 1); (2) the potential risks of participation; (3) safety arrangements during and after the study phase; (4) that TCOY cannot replace face-to-face therapy for alcohol misuse and (5) the circumstances under which they should contact their general practitioner (GP) or a professional from a medical advisory and emergency list that will be accessible at all times via 
Figure 1 Trial flow chart. This figure provides an overview of the participant flow for this trial.

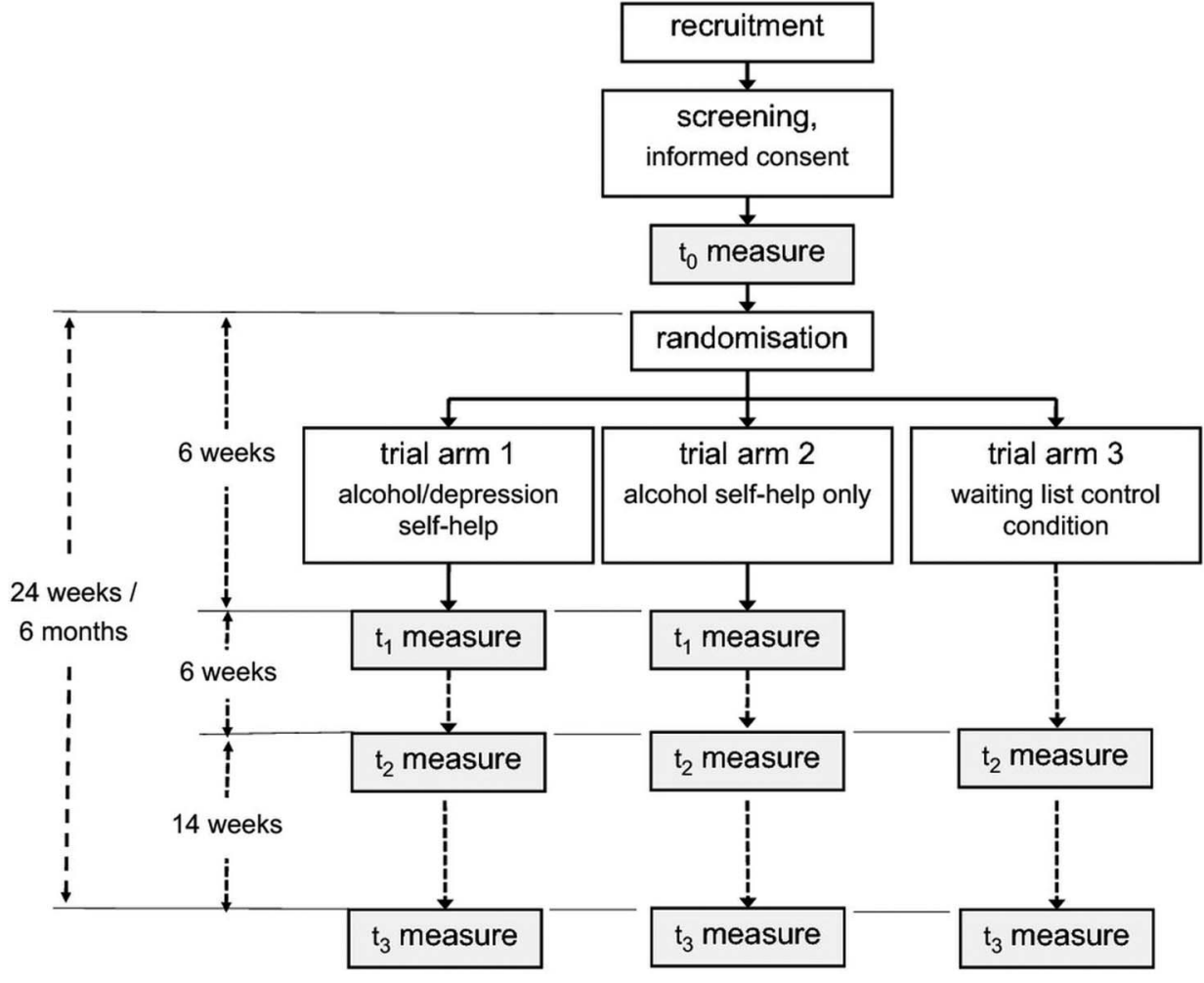

a menu item 'Help me'. Access will be granted to participants during and after the study participation until the 6 months follow-up, regardless of whether they withdraw or drop out from the study. Potential participants who screen positive for suicidal ideations or plans are excluded from the study (see table 1) and will be referred to their family doctor for additional screening or help by a staff member. The participants will also be informed of the approval of the study by the corresponding ethics committee in each country. Moreover, they will be informed of their right to withdraw from the study at any time without consequences.

Table 1 Inclusion and exclusion criteria and rationale

Inclusion criteria Reasoning

(1) Minimal age of 18 years

(2) AUDIT score $\geq 8$ (and in the Netherlands: an AUDIT score $<20$ )

(3) To have a CES-D-20 score $\geq 16$ and $\leq 32$

(4) To have at least weekly internet access

Exclusion criteria

(1) Participation in other psychosocial or pharmacological treatments for the reduction/cessation of alcohol use or the reduction of depression symptoms

(2) Use of opioids, cocaine or amphetamine-type stimulants in the past 30 days and/or cannabis use for more than three times a week in the previous 30 days (2) Ever been in treatment for cardiovascular problems

(3) Suicide attempt history, actual suicide plans, elevated probability for a suicide attempt (P4 suicidal screener score 'minimal risk')

(4) For female participants: pregnancy or breastfeeding
To ensure a minimum age of participation

To include alcohol users with at least harmful or hazardous alcohol use (and in the Netherlands, to exclude alcohol users likely to meet dependence criteria)

To include participants with at least significant or mild depression symptoms

To ensure minimal access to intervention

Reasoning

To avoid confounding treatment effects

To avoid confounding drug effects

To avoid the possibility of participants with these problems entering the study

To avoid the possibility of participants with these problems entering the study

To avoid the possibility of women with these conditions entering the study

AUDIT, Alcohol Use Disorder Identification Test; CES-D, Centre of Epidemiologic Studies of Depression Scale. 
The registration for a personal online account involves providing a nickname, email address, sociodemographic data and informed consent. In Switzerland and Germany, informed consent is given online by ticking check boxes, while in the Netherlands users are instructed to print and sign by hand the informed consent form and send it to the country-specific study coordinator. After submitting the online registration, the system sends an email with a validation link to confirm the user's email address. After clicking that link, they have to set a password which completes the registration process. The AUDIT and the Centre of Epidemiologic Studies of Depression Scale (CES-D-20) are the main instruments for the participant screening. Those who fulfil the ordinary criteria (AUDIT $\geq 8$ (and in the Netherlands <20) and CES-D-20 $\geq 16$ and $\leq 32$, see table 1) will take part in the regular study. Those who do not may still fulfil the criteria for one of two substudies with different consent procedures. The first substudy is for those with an AUDIT $\geq 8$ and a CES-D $<16$ and will compare the alcohol-only intervention with the waiting list control condition. The second is for those with an AUDIT $<8$ and a CES-D $\geq 16$ and will evaluate a transdiagnostic intervention for subthreshold depression. Participants who do not meet any of those requirements will be given notice that they cannot take part in the study, but can use the programme on their own. Additionally, they will be encouraged to seek help from local counselling services.

\section{Randomisation and trial flow}

Figure 1 provides an overview of the trial flow. If a participant successfully completes the baseline assessment (T0), he or she will be randomised and introduced to the corresponding study arm (study arms 1 and 2), or informed that they will be provided access to the intervention of study arm 1 after 6 months (waiting list condition, arm 3). A computer program will automatically randomise the participants for each country at a 1:1:1 ratio to 1 of 3 parallel groups, if they meet the requirements of the main study. If they only meet the requirements of the substudy, they will instead be automatically assigned at a 1:1 ratio to 2 of the 3 parallel groups. The randomisation process is partially restricted, in the sense that the same IP is assigned to the same group for the rest of the day, in order to prevent circumvention of the assigned group by registering multiple accounts.

At the 3 and 6 months follow-up (T2, T3), a research assistant will try to assess as many participants as possible, regardless of how long they remained in their study arm or on the particular study arm they were allocated to. Follow-up assessment will be performed in three steps. First, participants will be invited up to three times with email reminders. Should participants fail to complete the follow-ups despite the reminder, they will be contacted personally and motivated to complete the follow-up instrument, and offered an interview with study collaborators (telephone numbers will be recorded in the consent procedure, as an integral part of the study, in case there are contact problems at the follow-up assessment). In case participants refuse the telephone interview, they will be offered an interview on the primary outcome only. If participants still refuse, they will be asked to provide reason(s) for their refusal, which will be documented. Apart from active withdrawal of informed consent, we do not maintain any specific criteria for dropouts. We learnt from the consumption diaries of earlier studies ${ }^{11-14}$ that a substantial number of participants take breaks for 2 or more weeks during the interventions (eg, due to holiday absences) and finish the intervention several weeks later.

\section{Intervention}

The internet-based interventions in study arms 1 and 2 contain eight modules based on $\mathrm{CBT}^{28}$ and $\mathrm{MI}$ approaches $^{29}$ to treat alcohol use disorders. Adherencefocused guidance in study arms 1 and 2 is mainly based on the supportive-accountability model of providing guidance in internet interventions ${ }^{30}$ and was implemented as in previous studies. ${ }^{31} 32$ The intervention in study arm 1 contains behavioural self-help exercises targeting depression symptoms, alcohol misuse ${ }^{33}$ and social problem solving. ${ }^{34}$ The elements targeting depression symptoms are based on evidence-based treatment modules that have been tested in a number of different settings, including the treatment of subthreshold depression, major depression and depression in people with chronic somatic conditions. ${ }^{19} 32$ 35-38 Study arm 2 consists of the self-help exercises on alcohol misuse only. Allocation of participants to study arms 1 and 2 is blinded. Participants in study arm 3 are placed on a waiting list, are not blinded for their allocation and form a control group that can access the intervention in study arm 1 after their 6 months follow-up assessment.

\section{Intervention modules}

The eight modules are offered as an internet-based selfhelp intervention (study arms 1 and 2) and are accessible after successful registration, providing informed consent, and completion of the baseline assessment. In case participants have questions regarding these modules or face problems, they can contact their personal e-coach during the 6 weeks of the intervention. After completing an introduction module, the starting page will display explanations on the alcohol consumption diary (study arms $1 / 2$ ) and the mood and activity diary (study arm 1), the modules and the inputs. Participants will be introduced to six companions who will support them and provide their own experiences to the module tasks, and they will learn about the weekly email reminder for programme continuation as a further means of support. All eight modules are freely accessible from the start under the menu item 'modules' (see figure 2). It is recommended that these modules should be worked through in the order they are presented, although any order is feasible. They 
Figure 2 This figure provides an overview of the Take Care of You main menu for the reduction of alcohol use and depression symptoms in alcohol misusers with co-occurring depression symptoms.

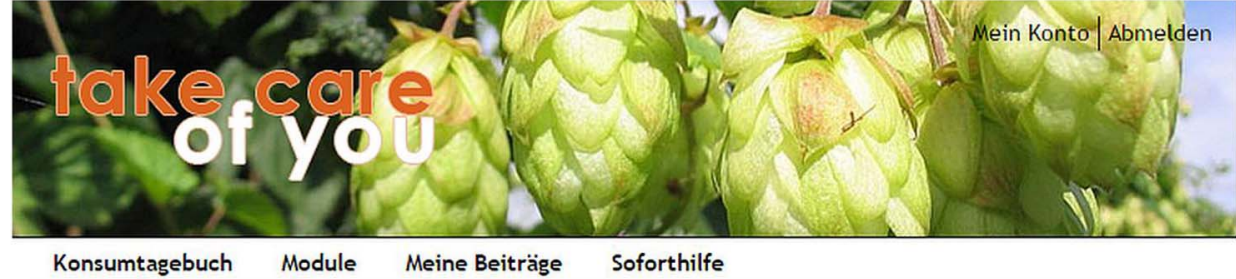

Konsumtagebuch Module Meine Beiträge Soforthilfe

\section{Module}

Hier sehen Sie die Übersicht aller 8 Module. Wir empfehlen Ihnen, jeweils pro Woche 1-2 Module durchzuführen, vorzugsweise in dieser Reihenfolge. Pro Modul wird lhr Fortschritt mit einem roten Balken am unteren Rand des Kästchens gekennzeichnet. Wenn Sie auf ein Modul klicken, fahren Sie auf der Seite weiter, wo Sie zuletzt waren.
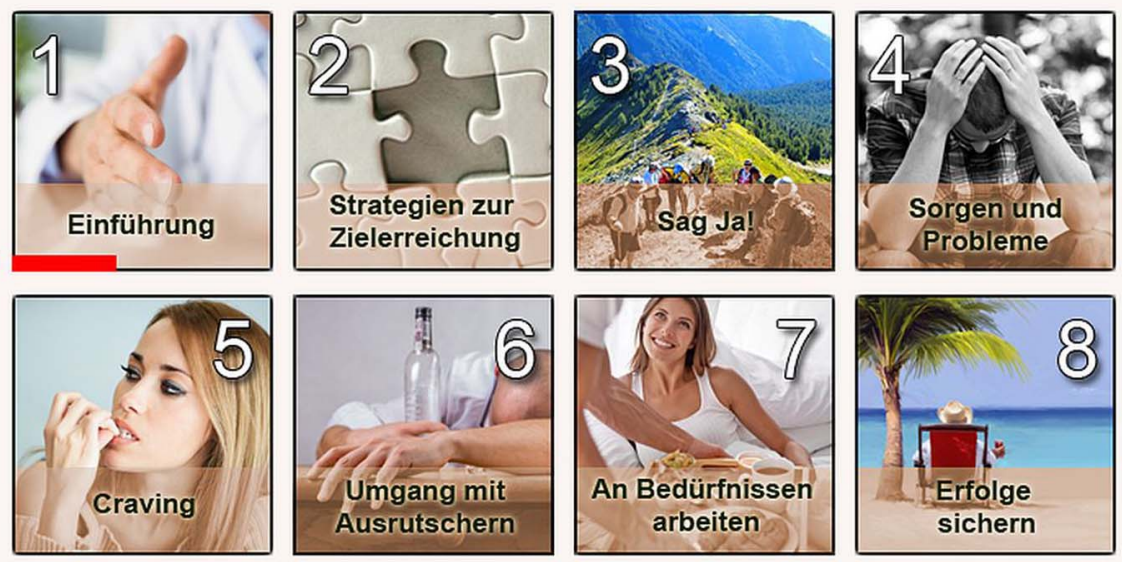

might, for instance, jump right to the craving module if they suffer a strong craving. Participants are recommended to repeat the modules as often as needed. The participants' progress is indicated with a progress bar for each module separately. If they leave a module (on purpose or accidentally) and revisit it later, they will automatically continue on the page they had left. They can always navigate forward and backward and jump back to the beginning of a module when they have finished it. The detailed structure of the eight modules in study arms 1 and 2 is presented in table 2 . In order to minimise module length as a possible confounding factor, we designed the corresponding modules to have the same approximate word count.

\section{Personal companion}

During the introduction, six possible fictional companions are briefly introduced. These are slightly different for study arms 1 and 2 (6 companion profiles each). Their function is to provide participants with situational advice and examples. They appear in each module at least once. The characters vary in sex, age, familial and professional situation, in order to provide a maximum of identification. At the start, all participants will choose the companion they can most identify with. That companion's statement will always be displayed first, but the statements of the other companions can still be browsed.
Forum

Participants in study arms 1 and 2 can also exchange messages in separate forums with other participants of their allocated study arm. Forum activities are recommended to increase the success of the intervention. A forum administrator with a clinical and health psychology background will moderate both forums, in order to check and to delete inappropriate contents.

\section{Adherence-focused guidance and intervention reminders}

The two active study arms 1 and 2 will include e-coaches to provide adherence-focused guidance. The purpose of the guidance is to support participants' adherence to the treatment modules while minimising the project team's expenditure. In line with the supportive accountability model, ${ }^{30}$ it is assumed that adherence to an internet-based intervention (and thus its effectiveness) can be enhanced by support from a coach who is seen as legitimate, trustworthy, benevolent and expert. The e-coach guidance will consist of two elements: (1) adherence monitoring and (2) feedback on demand. Adherence monitoring will include offering participants support in adhering to the intervention by regularly checking whether they have completed intervention sessions on time and, if not, by reminding them to do so and to reinforce their adherence. Both personal and automatic reminders have been shown to improve adherence to self-guided health promotion and 
Table 2 Module contents and comparison between alcohol and depression self-help (arm 1) and alcohol self-help only (arm 2)

\begin{tabular}{|c|c|}
\hline Jo & ssion self-help \\
\hline $\begin{array}{l}\text { M1 } \\
\text { mandatory }\end{array}$ & $\begin{array}{l}\text { Introduction ( } 1750 \text { words) } \\
\text { Introductory words (tailored to arm 1) } \\
\text { Pro and contra of drinking } \\
\text { Core motive for change } \\
\text { Confidence of change } \\
\text { - Introduction to consumption diary, mood barometer and } \\
\text { planning of positive activities }\end{array}$ \\
\hline M2 & $\begin{array}{l}\text { Strategies for goal achievement ( } 2370 \text { words) } \\
\text { Introduction ( } 80 \text { words) } \\
\text { Changing habits ( } 500 \text { words) } \\
\text { Alcohol at home ( } 860 \text { words) } \\
\text { - Exercise: resisting alcohol in specific situations for the } \\
\text { dimension availability and mood } \\
\text { Reflections on mood } \\
\text { Drinking in positive situations } \\
\text { Drinking in negative situations } \\
\text { Thanks, I don't drink (160 words) } \\
\text { Alcohol for relaxation } \\
\text { (Short version without link to M7) } \\
\text { My personal strategies (tailored to arm 1) }\end{array}$ \\
\hline M3 & $\begin{array}{l}\text { Say yes ( } 2330 \text { words) } \\
\text { - Positive activities ( } 1400 \text { words) } \\
\text { - Checkbox list with around } 80 \text { activities of } 5 \text { categories } \\
\text { - Incial, leisure time, relaxation, creativity, sports, culture } \\
\text { Common problems ( } 930 \text { words) } \\
\text { - Tips for motivational problems } \\
\text { - Tips for implementation problems }\end{array}$ \\
\hline
\end{tabular}

M4

Worries and problems (1850 words)

- Relation of depression and problems

- 6-step plan

- My problem

O Controllable problem

O Uncontrollable problem

- My goal?

- Possible solutions

- Planning of route to goal

- Try out the route

- Review and taking stock

M5 Craving (1300 words)

- Forms of craving (physical and mental)

- Craving and conditioned triggers

- How to handle craving

- Distract yourself

- Talk about it with someone

- Just be aware of it

- Bring to mind all negative consequences of consumption

- Talk to yourself

M6 Dealing with slips (474 words)

- Define what you consider a slip

- How to deal with it

- Plan your reaction for future slips
Arm 2: alcohol self-help only

Introduction (1700 words)

- Introductory words (tailored to arm 2)

- Pro and contra of drinking (same as arm 1)

- Core motive for change (same as arm 1)

- Confidence of change (same as arm 1)

- Introduction to consumption diary

Strategies for goal achievement (2825 words)

- Introduction (same as arm 1)

- Changing habits (same as arm 1)

- Alcohol at home (same as arm 1)

- Exercise: resisting alcohol in specific situations for the dimension availability and mood

- Reflection on availability

Alcohol at home: no good for me

Alcohol at home: no issue for me

- Alcohol for relaxation

(Short version with link to M7-PMR)

- My personal strategies (tailored to arm 2)

Say no (2630 words)

- Thanks, I don't drink (1700 words)

- Difficulties with rejecting offered alcohol (impolite, shame, outsider, problem outing)

- Exercise saying no

- Strategies against 'persistent seducers'

- Role-playing exercises

- Common problems (930 words)

- Tips for motivational problems

- Tips for implementation problems Identify risk situations (1550 words)

- Identify risk situations

- In negative emotions

- In positive emotions

- For relief/relaxation

- Habits

What now?

- Seemingly unimportant decisions

Craving (same as arm 1)

Dealing with slips (same as arm 1) 


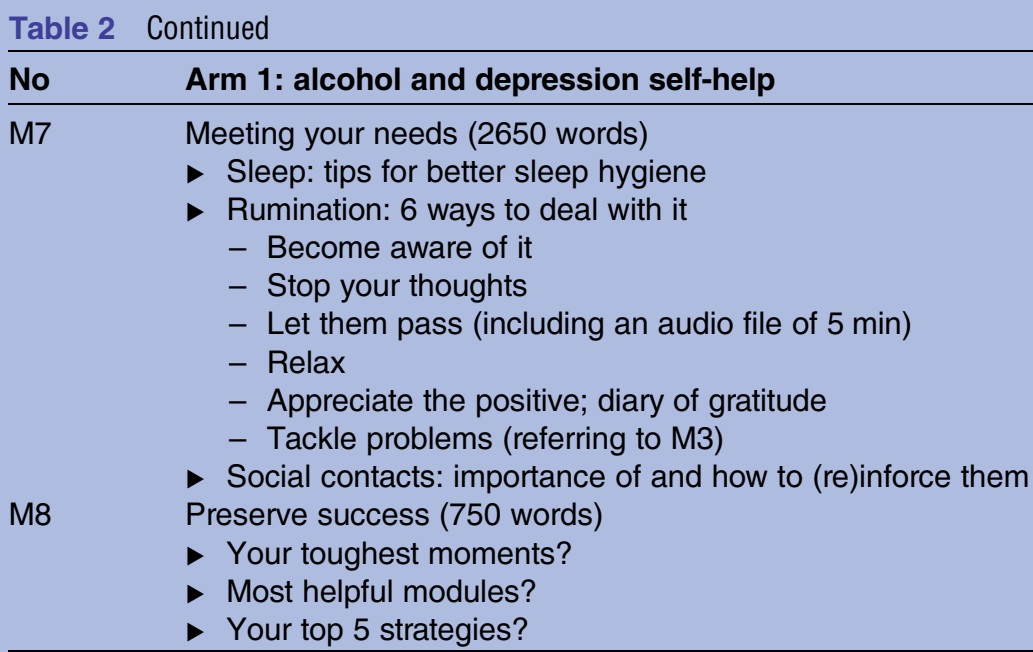

$F A Q$, frequently asked questions; PMR, progressive muscle relaxation. behaviour change interventions, ${ }^{39} 40$ but it is assumed that personal rather than automatic reminders from a coach are perceived as being more benevolent and more effective. Feedback on demand provides the participants with an opportunity to contact the coach via the platform or a standard email and to receive individual support/feedback whenever such a need develops. In contrast to other guidance concepts, the guidance is provided only when requested by the participants. Feedback is not assumed to have a direct influence on the effectiveness of the intervention. However, it aims to enhance the perception of the coach's legitimacy and the sense that the coach has the participant's best interests at heart. ${ }^{41}$ Individuals are assumed to respond more positively to adherence demands from a coach who is perceived as legitimate. ${ }^{30}{ }^{41}$ Hence, the perceived legitimacy of the coach is believed to enhance adherence to the intervention and to constitute a necessary requirement if adherence monitoring is to have a positive influence on the effectiveness of the intervention.

There are five types of emails that will be sent to the participants: (1) weekly emails, (2) diary feedback, (3) diary reminder, (4) follow-up invitations and (5) personal progression feedback. All emails are only sent to intervention arms 1 and 2, except for the follow-up invitations. (1) Weekly emails are automatically sent on the last day of each week. Their function is to accompany the participants through the 6 weeks with encouraging words, and to remind them to fill out the diary on the next day and sometimes to suggest using a particular module. (2) Diary feedback is automatically sent 2 days after the beginning of every week, if a participant has filled out his or her diary. Its content is a very short sentence and depends on the change in consumption compared with the previous week. The sentence is taken from a pool of $3 \times 6$ messages and can be positive (in case of a decrease in consumption), neutral (in case of stagnation) or compassionate (in case of an increase in consumption). (3) Diary reminders are automatically sent if the participant has not yet filled out his or her diary for 1 and/or 3 days after starting the new week. (4) Follow-up invitations are automatically sent on the day when the person is meant to fill out a questionnaire. This is the only email that will also be sent to the waiting list. (5) Personal progression feedback is the only type of email that is sent manually. The first one is a standard email sent in the first week, in which the e-coach introduces himself or herself, with an offer of contact if there are questions or problems during the 6 weeks of study participation. Further emails are sent manually in predefined circumstances. There are two main types of circumstances when such emails are sent-first, when participants progress too slowly or too rapidly through the modules, and second, when participants have given certain answers in particular modules. In the latter case, particular suggestions are provided. For example, if a participant has ticked that he/she often drinks alcohol when 'invited for drinks', 'after work with colleagues' or 'when alcohol was available for free' in the module 'identify risk situations', the e-coach can suggest that he/she should look into the module 'Say no!', if he/she has not yet done so. For easy administration, the e-coach will be given online access to several customised user lists showing only participants who have ticked off those answers and the page they have reached in the module we wish to recommend. The email itself has to be triggered and entered manually, but can be sent to multiple users from the administration backend.

\section{Technical specifications}

TCOY is a website that is based on Drupal 7 and incorporates the self-help parts of a previously implemented design. ${ }^{11-14}$ The website has a responsive design and automatically adapts to tablets and smartphones. Participants are informed that they can also use their mobile devices. Any internet user can register an online account via one of the country-specific internet websites of TCOY (http://www.takecareofyou.nl, http://www. 
takecareofyou.de, http://www.takecareofyou.ch), by providing a unique username and email address. These three websites are technically independent installations with separate databases and user management. To prevent the creation of accounts with invalid email addresses, the registration process will not be completed until a verification email link has been clicked and a personal password has been set. To prevent evasion of the assigned group by registering a second account, we have implemented a mechanism to assign users with the same IP to the same group for the rest of the day.

\section{Waiting list condition}

Participants randomised to the waiting list will have the opportunity to participate in the internet-based intervention (study arm 1) 6 months after registration. Healthcare utilisation will be monitored closely in all groups.

\section{Measurements}

Table 3 presents an overview of the measurements used at baseline, as well as after 6 weeks in treatment and after 3 and 6 months follow-up.

The primary outcome measure comprises the quantity of alcohol use in the previous 7 days in number of standard drinks and will be assessed by timeline follow-back, a widely used measure in studies aiming at controlled drinking (timeline followback; TLFB) ${ }^{42}$

The secondary outcome measures consist of the following measures: (1) the total score of the short version of AUDIT-C. ${ }^{43}$ We will also assess the full second edition of the $\operatorname{AUDIT}^{4}$ assessment at baseline, for a more comprehensive screening of alcohol use disorders and for comparisons with participants of other studies. An AUDIT score of $\geq 8$ is used to identify harmful or hazardous alcohol use in both sexes. (2) The CES-D-20. ${ }^{44}$
The CES-D scale is a short self-report scale designed to measure depression symptoms in the general population. ${ }^{44} 45$ The items of the scale are symptoms associated with depression that have been used in previously validated longer scales. The CES-D scale has been tested in household interview surveys and in psychiatric settings and found to have very high internal consistency and adequate test-retest reliability. CES-D responses are based on the frequency of occurrence of depression symptoms during the past week. It uses a four-point ordinal scale: rarely or none of the time $(<1$ day); some or a little of the time (1-2 days); occasionally or a moderate amount of the time (3-4 days); most or all of the time (5-7 days). The scores of the CES-D-20 range between 0 and 60. A CES-D-20 cut-off score of 16 is indicative of 'significant' or 'mild' depression symptoms. It is equivalent to experiencing six symptoms for most of the previous week or a majority of symptoms on 1 or 2 days. Higher scores indicate a higher symptom load. In order to prevent individuals with more severe depression symptoms entering the study, participants with a CES-20 score higher than 32 will be excluded from the sample after the study but offered access to the intervention and recommended additional face-to-face treatment. (3) A combined alcohol and depression symptoms measure, falling below the AUDIT-C cut-off of 4 in women and 5 in men (no more severe alcohol use disorder according to the Diagnostic and Statistical Manual of Mental Disorders, Fifth Edition (DSM-V) $)^{46}$ and the CES-D-20 cut-off of 16 (no more significant or mild depression symptoms). This measure is calculated during the intervention analyses. (4) The number of alcohol-free days per week assessed by TLFB. ${ }^{42}$ (5) The 'Fragebogen Substanzanamnese' (FDA) that ascertains the years of lifetime consumption, the past month's consumption,

Table 3 Measurements and instruments

\begin{tabular}{|c|c|c|c|c|}
\hline Assessments/instruments & Baseline & 6 weeks & 3-month follow-up & 6-month follow-up \\
\hline Sociodemographics & $x$ & & & \\
\hline FDA & $x$ & & & \\
\hline AUDIT & $x$ & & & \\
\hline AUDIT-C & $\mathrm{x}$ & & $\mathrm{x}$ & $\mathrm{x}$ \\
\hline CES-D-20 & $\mathrm{x}$ & & $\mathrm{x}$ & $\mathrm{x}$ \\
\hline AUDIT-C $<4$ in women and $<5$ in men and CES-D-20 $<16$ & $x$ & & $\mathrm{x}$ & $\mathrm{x}$ \\
\hline Number of consumption free days* & $x$ & & $\mathrm{x}$ & $\mathrm{x}$ \\
\hline Number of weekly standard drinks* & $\mathrm{x}$ & & $\mathrm{x}$ & $\mathrm{x}$ \\
\hline MHI-5 & $x$ & & $x$ & $x$ \\
\hline Suicidal ideations or plans & $x$ & & $x$ & $x$ \\
\hline EQ-5D-5L & $x$ & & $x$ & $x$ \\
\hline TiC-P & $x$ & & $x$ & $x$ \\
\hline WAI presenteeism & $x$ & & $x$ & $x$ \\
\hline ZUF-8† & & $x$ & & \\
\hline
\end{tabular}


and the manner of consumption for the DSM-IV/ International Classification of Diseases (ICD)-10 substances of abuse. This measure was derived from the EuropeASI. ${ }^{47}$ (6) The short version of the Mental Health Inventory (MHI-5) ${ }^{48}$ This is a validated and userfriendly self-assessment questionnaire that assesses recent mental distress and self-reported diagnoses of depression. In addition, we will also ask participants to indicate any suicidal or self-harm ideations or plans at the follow-ups. (7) The quality of life is measured by the five-level variant of the five-dimensional EuroQol instrument (EQ-5D-5L), a standardised instrument used as a measure of health outcome that provides a simple descriptive profile and a single index value for health status. $^{49}$ (8) Direct medical and direct non-medical cost data are collected with the Trimbos and iMTA questionnaire on 'Costs associated with Psychiatric Illness' (TiC-P).$^{50}$ (9) Work ability is measured by the single item Work Ability Index (WAI). ${ }^{51}$ (10) Treatment retention is calculated as the percentage of completed weekly consumption diary entries (study arms 1 and 2). (11) The German client satisfaction questionnaire ZUF-8 has been extensively studied, and is easily scored by summing the individual item scores to produce a range of 8-32, with high scores indicating greater satisfaction. $^{52} 53$ In study arms 1 and 2, participation in the intervention as an indicator of treatment adherence will be measured through diary entries, the number of completed modules, and the number of logins in the login history between the baseline assessment and the 6-month follow-up. (12) Lastly, the occurrence of any negative effects will be identified as in Rozental et $a \tilde{l}^{4}$ at the 6 months follow-up.

German and Dutch validations are available for all outcome instruments. Unit resource use (GP visits, ill days, etc) will be multiplied by their appropriate integral country-specific cost prices for the TiC-P.

\section{Sample size calculation}

An estimation of the effect sizes is necessary because of a lack of comparable internet-based self-help studies that address both problematic alcohol use and depression symptoms. On the basis of an expert opinion, we can postulate a small effect size with Cohen's $d=0.25$ for the reduction in the weekly number of standard drinks between study arms 1 and 3 at 6 months postrandomisation. We aim to include 756 participants for all three countries and study arms, 100 in the Netherlands and 328 each in Switzerland and Germany. This sample size will allow us to detect an effect size of $d=0.25$ based on a power $(1-\beta)$ of $80 \%$ and an $\alpha$ error of 0.05 in a twosided test, calculated using $G^{*}$ Power software.

\section{Data analyses}

Data will be analysed according to the intention-to-treat (ITT) principle. To address missing data for the ITT analyses, we will apply multiple imputation procedures of R (R Foundation for Statistical Computing, Vienna) with Amelia-2. Differences between study arms in primary and secondary continuous outcome variables at baseline and the follow-up points will be tested using (robust/generalised) linear mixed models (LMMs). LMMs will be specified appropriately to model clusters and repeated measures by defining random effects for study arms and time (repeated measures). For nonnormal continuous outcomes, appropriate distributions (eg, negative binomial, zero-inflated) will be specified. ${ }^{55}$ Appropriate covariance matrices for the random effects will be used. For binary outcomes, a generalised LMM (GLMM) will be specified that defines an appropriate link function. In the GLMM fixed effect, coefficients will be interpreted in the context of the participant-specific (non-marginal) model fit. In order to investigate the exploratory research question as to whether combined treatment for depression and alcohol results in a similar reduction in alcohol consumption as with alcohol treatment alone, a CI approach will be used for the effect size of the difference between the two study arms, with a two-sided 0.05 level of significance. ${ }^{56}$ The equivalence margin is a priori set at $\mathrm{d}=0.20$, corresponding to the smallest value that would present a relevant effect. ${ }^{57}$ The upper bound of the $95 \%$ CI for the effect size will be compared with the predefined equivalence margin of $\mathrm{d}=0.20$ and will have to be below the margin to show equivalence. In addition to the ITT analyses, perprotocol analyses will be performed.

\section{Economic evaluation \\ Costs}

We will consider four types of costs: (1) the costs of offering the TCOY interventions, (2) costs stemming from general healthcare uptake besides the TCOY interventions, including the costs of medication, (3) patients' out-of-pocket expenses (eg, leisure time spent on receiving care) and (4) costs stemming from productivity losses due to absenteeism or reduced efficiency while at work (presenteeism). The first two types of costs are also known as the direct medical costs and these will be based on the full economic costs of offering the interventions. Here, we will use national guidelines for economic evaluation (ie, for the Netherlands; ${ }^{50}$ for Germany and also for Switzerland as there are none for Switzerland), ${ }^{58} 59$ and rely on the standard cost prices reported therein. Productivity losses will be based on the sex-specific and age-specific labour costs for each of the three countries. Data on resource use (healthcare uptake) and productivity losses will be collected with the widely used TiC-P. ${ }^{60}$

\section{Effects}

As an effect measure, the EQ-5D-5 $\mathrm{L}^{61}$ will be used to compute health gains expressed in quality-adjusted life years (QALYs). Health utilities are a major feature of the EQ-5D instrument, and are available for the Netherlands ${ }^{62}$ and Germany ${ }^{63}$ For Switzerland, the German tariffs will be used as there are no EuroQol-recognised German-Swiss 
health utilities for the EQ-5D available. Since the EQ-5D-5L is a recent update of the three-level EQ-5D, studies that directly elicit preferences from general population samples to derive value sets to calculate the EQ-5D-5L health utilities are currently under development (EuroQol group 2015) ${ }^{64}$ In the meantime, the EuroQol Group has coordinated a study that administered both the three-level and five-level versions of the EQ-5D, in order to develop a 'crosswalk' between the EQ-5D-3L and the EQ-5D-5L. This has resulted in crosswalk value sets for the EQ-5D-5L. ${ }^{65}$ It is expected that the new utilities for the EQ-5D-5L will be available before we perform the economic evaluations for the current project; otherwise, the crosswalk from the EuroQol Group will be used to calculate health utilities.

\section{Cost-effectiveness calculations}

The economic evaluation will be conducted alongside the randomised trial, taking into account the CHEERS statement ${ }^{66}$ and the 2015 ISPOR good research practices task force report on cost-effectiveness analysis alongside clinical trials. ${ }^{67}$ From the technical point of view, the economic evaluation referred to as cost-effectiveness analysis in the following section will be both a costeffectiveness analysis and a cost-utility analysis. For the cost-effectiveness analysis, positive treatment response, a dichotomous outcome measure, is defined as falling below the AUDIT-C cut-off for severe alcohol use disorder and the CES-D-20 cut-off of 16 (no more significant or mild depression symptoms). For the cost-utility analysis, health utilities based on the EQ-5D instrument will be used as the effect measure.

Using the area under the curve method, the periods between the measurement waves will be weighted by the utility of the health state in that period. This allows computation of QALYs over the entire trial period (24 weeks between randomisation and the last follow-up wave) for the cost-utility analysis. In a similar vein, cumulative costs over the entire follow-up period will be obtained from the cost estimates at the various measurement waves. The cost-effectiveness and cost-utility evaluations will be performed in line with suggestions by Drummond et al, ${ }^{68}$ that is, in agreement with the ITT principle, with missing data addressed using imputation.

The incremental cost-effectiveness ratio (ICER) will be calculated as follows: ICER $=(\mathrm{C} 1-\mathrm{C} 2) /(\mathrm{E} 1-\mathrm{E} 2)$, where C stands for costs, E for effects (either the average change in QALYs or the proportion of participants meeting criteria for positive treatment response), and subscripts 1 and 2 refer to the two trial arms compared. Since this study comprises three trial arms, three such comparisons will be analysed and presented. CIs around the ICER will be calculated using a non-parametric bootstrap approach: 5000 non-parametric bootstrapped samples will be extracted from the original data set. For each of these bootstrapped samples, the incremental costs, incremental effects and the ICER will be calculated for each comparison between two of the three trial arms separately. The resulting 5000 ICERs will be used for further calculations and will be plotted on a costeffectiveness plane. In addition, cost-effectiveness acceptability curves (CEACs) will be constructed. One-way sensitivity analyses directed at uncertainty in the main cost drivers will be performed to gauge the robustness of our findings. In addition, a sensitivity analysis in which a covariate-adjusted CEAC curve is constructed will be conducted using net benefit regression methods. ${ }^{69} 70$

\section{Ethics and dissemination}

The ethics committee of Leuphana forewent a separate ethics procedure, as the study had already been approved in Switzerland. The trial is registered at Current Controlled Trials and traceable as ISRCTN10323951.

Results will be reported at conferences and in peerreviewed publications. A participant-friendly summary of trial findings will be published on the TCOY websites in each participating country.

\section{DISCUSSION}

To the best of our knowledge, this will be the first RCT to compare the efficacy and cost-effectiveness of an internet-based self-help intervention with adherencefocused guidance for alcohol misuse and depression symptoms, in comparison to internet-based self-help with adherence-focused guidance for problematic drinking only in an at-risk general population sample. The expected findings will extend our insights in designing effective internet-based interventions in general and more specifically for comorbid alcohol use disorder and depression. In particular, this easy to access and anonymous intervention may be of special benefit for otherwise well-integrated individuals who suffer from depression symptoms and fear stigmatisation for their alcohol misuse and who therefore fail to visit addiction counselling and treatment centres.

To the best of our knowledge, there are no available data on comparative cost-effectiveness studies of internet-based indicated prevention or dual-disorder treatments of alcohol and depression versus alcohol treatment alone.

Since the objective is to reduce alcohol misuse and depression symptoms, it makes sense to tackle both problems in the same self-help intervention and some underlying theory is explicitly introduced in the combined alcohol and depression self-help intervention. However, it was challenging to compile and develop this combined intervention. First, we decided to follow the results of the meta-analysis of Riper $e t a l^{25}$ that looked at face-to-face interventions. This meta-analysis only focused on studies that included CBT and MI, since these therapeutic approaches have been shown to be superior to others in previous studies on the treatment of comorbid alcohol use disorder and depression. ${ }^{71}$ This meta-analysis implies that a combined intervention has to include rather more modules addressing problematic 
drinking than depression symptoms and that integrated interventions do not perform better than interventions including modules addressing one problem after the other. Second, we had to adjust this combined intervention to the lengths of the alcohol self-help only study arm, in order to avoid the possibility that intervention length has an influence on outcomes of the two respective study arms. Third, we decided that the maximal number of modules to be included was eight. Moreover, we aimed for concise modules in order to avoid early dropouts after successful study registration. Thus, the content of the combined alcohol and depression intervention (study arm 1) is sometimes integrated (eg, reflections on mood in module 2, strategies for goal achievements) and sometimes replaced with a completely new depression module (eg, module 3 on activity planning replaced module 3 of the alcohol only intervention that aims at fostering alcohol-related refusal skills). Moreover, some modules were shortened to reach comparable intervention lengths without losing relevant concepts (eg, module 3 of study arm 2 that aims at fostering alcohol-related refusal skills was shortened and inserted into module 2 of study arm 1). However, this study allows us to answer the question as to whether an internet-based intervention to target two inter-related mental health problems (problematic alcohol use and depression symptoms)-either integrated or consecutive-is better than an intervention of comparable length addressing alcohol use problems only. Moreover, we expect the planned study to extend our knowledge as to whether integrated dual diagnosis interventions lead to higher intervention satisfaction than treatment interventions without explicit focus on a dual diagnosis. ${ }^{27}$ One of the advantages of TCOY is its responsive web design that also allows mobile phone participation. We hope that participants will more frequently fill out their consumption and mood diary and potentially provide diary entries that are more valid and not too dependent on retrospective distortions. On the other hand, we expect that distraction during longer and more complex tasks will be rather high when participants are on the move. Therefore, we also recommend that participants should take enough time-at least once a week-to complete their modules in a calm place.

Aside from the large sample size needed for the alcohol and depression group, there are other reasons why we sought collaboration in multiple countries, even from the early stage of the TCOY project. Disease and disability are not stopped by national borders and especially not limited to borders when delivered through the internet. In addition, participation rates may depend on the distance to the nearest mental health centre. For example, for rural areas in Switzerland, these distances will be higher and may result in a greater treatment adherence to TCOY, whereas in a densely populated country such as the Netherlands, there may be more rapid transfer from the TCOY intervention to local mental healthcare.

\section{Limitations}

This study has the following limitations. First, the exclusion of people who are currently receiving psychosocial or pharmacological treatments to support reducing or quitting alcohol use and depression symptoms is an important limitation. However, this study aims at testing the efficacy of an internet-based self-help intervention for people with co-occurring problematic alcohol use and depression symptoms who are not likely to seek traditional treatment services due to stigma, anxiety or a lack of time resources.

Second, owing to limitations with regard to feasibility, only self-reported measurements will be used. Third, most of the self-report instruments show good psychometric properties, but only a few have been validated in the context of online assessments, for example, the TLFB. $^{42}$ Finally, the expected high attrition rates in study arms 1 and 2 during the 6 weeks of the interventions will be a major limitation of this study. We will address this issue in four ways: (1) all participants will be asked to invest $\sim 25 \mathrm{~min}$ on the baseline assessment, which may select more motivated participants and prevent participation of unmotivated participants; (2) participants will be sent adherence-focused weekly motivational reminder emails; (3) we have integrated fictional personal companions and have provided the users with the possibility to share their personal intervention progress and experiences in a forum with their participating peers, in order to increase personal commitment. Last but not least, all missing values in the final data set will be imputed for ITT analyses in addition to perprotocol analyses, as we expect high levels of study dropouts at the follow-ups and want to estimate the sensitivity of our findings to the approach chosen for missing data.

\section{CONCLUSION}

This study allows one to assess the (cost-)effectiveness of an internet-based intervention for reducing co-occurring alcohol misuse and depression symptoms simultaneously. If shown to be effective, the TCOY intervention is a valuable means to reach people in the general population who do not use traditional mental healthcare and addiction counselling services.

\section{Author affiliations}

${ }^{1}$ Swiss Research Institute for Public Health and Addiction, University of Zurich, Zurich, Switzerland

${ }^{2}$ Department of Research, Arkin Mental Health Care, Amsterdam, The Netherlands

${ }^{3}$ Department of Psychiatry, Academic Medical Centre, University of Amsterdam, Amsterdam, The Netherlands

${ }^{4}$ Department of Health Psychology and Applied Biological Psychology, Leuphana University Lueneburg, Lueneburg, Germany

${ }^{5}$ Division of Health Training Online, Innovation Incubator, Leuphana University Lueneburg, Lueneburg, Germany

${ }^{6}$ Department of Clinical Psychology, Vrije Universiteit Amsterdam, Amsterdam, The Netherlands

${ }^{7}$ Clinical Psychology and Psychotherapy, Friedrich-Alexander University Erlangen Nuremberg, Erlangen, Germany 
Twitter Follow Heleen Riper at @heleenriper

Acknowledgements This study is supported by the Swiss Foundation for Alcohol Research grant number 247.

Contributors MPS and MB had the initial idea for this study. MPS, MB and DDE prepared the first draft of the paper and MPS the final manuscript. MPS, $M B, D L, M D, A W, L J M$ and DDE developed the intervention of study arms 1 and 2. AW programmed and implemented the study websites of TCOY. HR, JD, AEG and SH provided continuous feedback on the development of the interventions and the present study protocol. LJM and MPS revised the first version of the study protocol thoroughly. All authors approved the final version of the manuscript submitted for publication.

Funding Swiss Foundation for Alcohol Research.

Competing interests DL and DDE are shareholders of GET.ON Institute, a company that provides internet interventions and aims to foster their implementation in routine care in Germany.

Ethics approval This RCT will be executed in compliance with the Helsinki Declaration and was approved by the ethics committee of the Canton of Zurich on 31 March 2015 (KEK-ZH-Nr. 2015-0082) and the medical ethics committee of the University of Amsterdam on 11 November 2015 (METc-VUmc-No. 2015.228).

Provenance and peer review Not commissioned; externally peer reviewed.

Data sharing statement Qualified researchers can ask for data sharing by the first author MPS after the study finalisation.

Open Access This is an Open Access article distributed in accordance with the Creative Commons Attribution Non Commercial (CC BY-NC 4.0) license, which permits others to distribute, remix, adapt, build upon this work noncommercially, and license their derivative works on different terms, provided the original work is properly cited and the use is non-commercial. See: http:// creativecommons.org/licenses/by-nc/4.0/

\section{REFERENCES}

1. WHO (2013). Draft comprehensive mental health action plan 20132020. http://bit.ly/Vhhh8s

2. Whiteford HA, Degenhardt L, Rehm J, et al. Global burden of disease attributable to mental and substance use disorders: findings from the Global Burden of Disease Study 2010. Lancet 2013;382:1575-86.

3. WHO (2014). Global status report on alcohol and health 2014. http:// apps.who.int/iris/bitstream/10665/112736/1/9789240692763 eng.pd

4. Saunders JB, Aasland OG, Babor TF, et al. Development of the Alcohol Use Disorders Identification Test (AUDIT): WHO Collaborative Project on Early Detection of Persons with Harmful Alcohol Consumption-II. Addiction 1993;88:791-804.

5. Conway KP, Compton W, Stinson FS, et al. Lifetime comorbidity of DSM-IV mood and anxiety disorders and specific drug use disorders: results from The National Epidemiologic Survey on Alcohol and Related Conditions. J Clin Psychiatry 2006;67:247-58.

6. Cranford JA, Nolen-Hoeksema S, Zucker RA. Alcohol involvement as a function of co-occurring alcohol use disorders and major depressive episode: Evidence from The National Epidemiologic Survey on Alcohol and Related Conditions. Drug Alcohol Depend 2011;117:145-51.

7. Stinson FS, Grant BF, Dawson DA, et al. Comorbidity between DSM-IV alcohol and specific drug use disorders in the United States: Results from The National Epidemiologic Survey on Alcohol and Related Conditions. Drug Alcohol Depend 2005;80:105-16.

8. Swendsen JD, Merikangas KR, Canino GJ, et al. The comorbidity of alcoholism with anxiety and depressive disorders in four geographic communities. Compr Psychiatry 1998;39:176-84.

9. Bott $\mathrm{K}$, Meyer $\mathrm{C}, \mathrm{Rumpf} \mathrm{HJ}$, et al. Psychiatric disorders among at-risk consumers of alcohol in the general population. J Stud Alcohol 2005;66:246-53.

10. van Zoonen K, Buntrock C, Ebert DD, et al. Preventing the onset of major depressive disorder: a meta-analytic review of psychological interventions. Int J Epidemiol 2014;43:318-29.

11. Schaub MP, Maier LJ, Wenger A, et al. Evaluating the efficacy of a web-based self-help intervention with and without chat counseling in reducing the cocaine use of problematic cocaine users: the study protocol of a pragmatic three-arm randomized controlled trial. BMC Psychiatry 2015;15:156.
12. Schaub M, Sullivan R, Haug S, et al. Web-based cognitive behavioral self-help intervention to reduce cocaine consumption in problematic cocaine users: randomized controlled trial. J Med Internet Res 2012;14:e166.

13. Schaub MP, Wenger A, Berg O, et al. A web-based self-help intervention with and without chat counseling to reduce cannabis use in problematic cannabis users: three-arm randomized controlled trial. J Med Internet Res 2015;17:e232

14. Schaub MP, Haug S, Wenger A, et al. Can reduce-the effects of chat-counseling and web-based self-help, web-based self-help alone and a waiting list control program on cannabis use in problematic cannabis users: a randomized controlled trial. BMC Psychiatry 2013;13:305.

15. Tait RJ, Spijkerman R, Riper H. Internet and computer based interventions for cannabis use: a meta-analysis. Drug Alcohol Depend 2013;133:295-304.

16. Tait RJ, McKetin R, Kay-Lambkin F, et al. A web-based intervention for users of amphetamine-type stimulants: 3-month outcomes of a randomized controlled trial. JMIR Ment Health 2014;1:e1.

17. Riper $\mathrm{H}$, Blankers $\mathrm{M}$, Hadiwijaya $\mathrm{H}$, et al. Effectiveness of guided and unguided low-intensity internet interventions for adult alcohol misuse: a meta-analysis. PLOS ONE 2014;9:e99912.

18. Riper H, Spek V, Boon B, et al. Effectiveness of e-self-help interventions for curbing adult problem drinking: a meta-analysis. J Med Internet Res 2011;13:e42.

19. Buntrock C, Ebert D, Lehr D, et al. Effectiveness of a web-based cognitive behavioural intervention for subthreshold depression: pragmatic randomised controlled trial. Psychother Psychosom 2015;84:348-58.

20. Balhara YP, Verma R. A review of web based interventions for managing tobacco use. Indian J Psychol Med 2014; 36:226-35.

21. Hedman E, Ljótsson B, Lindefors N. Cognitive behavior therapy via the internet: a systematic review of applications, clinical efficacy and cost-effectiveness. Expert Rev Pharmacoecon Outcomes Res 2012;12:745-64.

22. Ebert DD, Zarski AC, Christensen $\mathrm{H}$, et al. Internet and computer-based cognitive behavioral therapy for anxiety and depression in youth: a meta-analysis of randomized controlled outcome trials. PLoS ONE 2015;10:e0119895.

23. Richards D, Richardson T. Computer-based psychological treatments for depression: a systematic review and meta-analysis. Clin Psychol Rev 2012;32:329-42.

24. Pettinati HM, O'Brien CP, Dundon WD. Current status of co-occurring mood and substance use disorders: a new therapeutic target. Am J Psychiatry 2013;170:23-30.

25. Riper $\mathrm{H}$, Andersson $\mathrm{G}$, Hunter SB, et al. Treatment of comorbid alcohol use disorders and depression with cognitive-behavioural therapy and motivational interviewing: a meta-analysis. Addiction 2014;109:394-406.

26. Schulte SJ, Meier PS, Stirling J. Dual diagnosis clients' treatment satisfaction-a systematic review. BMC Psychiatry 2011;11:64.

27. Deady M, Mills KL, Teesson M, et al. An online intervention for co-occurring depression and problematic alcohol use in young people: primary outcomes from a randomized controlled trial. J Med Internet Res 2016;18:e71.

28. Marlatt GA. Relapse prevention: maintenance strategies in treatment of addictive behaviors. New York: Guildford, 1985

29. Miller WR, Rollnick S. Motivational interviewing: helping people change. 3rd edn. New York: Guilford Press, 2012.

30. Mohr DC, Cuijpers P, Lehman K. Supportive accountability: a model for providing human support to enhance adherence to eHealth interventions. J Med Internet Res 2011;13:e30.

31. Boss L, Lehr D, Berking M, et al. Evaluating the (cost-)effectiveness of guided and unguided internet-based self-help for problematic alcohol use in employees-a three arm randomized controlled trial. BMC Public Health 2015;15:2224.

32. Ebert D, Lehr D, Smit F, et al. Efficacy and cost-effectiveness of minimal guided and unguided internet-based mobile supported stress-management in employees with occupational stress: a three-armed randomised controlled trial. BMC Public Health 2014; $14: 807$.

33. Lewinsohn PM. The behavioral study and treatment of depression. In: Hersen M, Eisler RM, Miller PM, eds. Progress in behavior modification. Vol 1. New York: Academic, 1975:19-65.

34. D'Zurilla TJ, Nezu AM. Social problem solving in adults. In: Kendall PC, ed. Advances in cognitive-behavioral research and therapy. Elsevier, 1982:201-74.

35. Buntrock C, Ebert DD, Lehr D, et al. Evaluating the efficacy and cost-effectiveness of web-based indicated prevention of major depression: design of a randomised controlled trial. BMC Psychiatry 2014;14:25. 
36. Ebert DD, Lehr D, Boß L, et al. Efficacy of an internet-based problem-solving training for teachers: results of a randomized controlled trial. Scand J Work Environ Health 2014;40:582-96.

37. Nobis S, Lehr D, Ebert D, et al. Efficacy and cost-effectiveness of a web-based intervention with mobile phone support to treat depressive symptoms in adults with diabetes mellitus type 1 and type 2: design of a randomised controlled trial. BMC Psychiatry 2013;13:306.

38. Nobis S, Lehr D, Ebert DD, et al. Efficacy of a web-based intervention with mobile phone support in treating depressive symptoms in adults with type 1 and type 2 diabetes: a randomized controlled trial. Dia Care 2015;38:776-83.

39. Fry JP, Neff RA. Periodic prompts and reminders in health promotion and health behavior interventions: systematic review. J Med Internet Res 2009;11:e16.

40. Titov N, Dear BF, Johnston L, et al. Improving adherence and clinical outcomes in self-guided internet treatment for anxiety and depression: randomised controlled trial. PLOS ONE 2013;8:e62873.

41. Tyler TR. Psychological perspectives on legitimacy and legitimation. Annu Rev Psychol 2006;57:375-400.

42. Sobell MB, Sobell LC. Problem drinkers: guided self-change treatment. New York: Guilford Press, 1993.

43. Bradley KA, DeBenedetti AF, Volk RJ, et al. AUDIT-C as a brief screen for alcohol misuse in primary care. Alcohol Clin Exp Res 2007;31:1208-17.

44. Radloff LS. The CES-D Scale: a self-report depression scale for research in the general population. Appl Psychol Meas 1977;1:385-401.

45. Riediger M, Linden M, Wilms HU. Die deutsche Version der CES-D als Instrument der gerontologischen Forschung. Z Klin Psychol Psychiatr Psychother 1998;46:344-64.

46. Dawson DA, Smith SM, Saha TD, et al. Comparative performance of the AUDIT-C in screening for DSM-IV and DSM-5 alcohol use disorders. Drug Alcohol Depend 2012;126:384-8.

47. Kokkevi A, Hartgers C. EuropASI: European adaptation of a multidimensional assessment instrument for drug and alcohol dependence. Eur Addict Res 1995;1:208-10.

48. Rumpf HJ, Meyer $\mathrm{C}$, Hapke U, et al. Screening for mental health: validity of the MHI-5 using DSM-IV Axis I psychiatric disorders as gold standard. Psychiatry Res 2001;105:243-53.

49. Dawson DA, Smith SM, Saha TD, et al. Comparative performance of the AUDIT-C in screening for DSM-IV and DSM-5 alcohol use disorders. Drug Alcohol Depend 2012;126:384-8.

50. van Roijen LH, van Straten A, Al Maiwenn, et al. Cost-utility of brief psychological treatment for depression and anxiety. $\mathrm{Br} J$ Psychiatry 2006;188:323-9.

51. Ahlstrom L, Grimby-Ekman A, Hagberg M, et al. The work ability index and single-item question: associations with sick leave, symptoms, and health-a prospective study of women on long-term sick leave. Scand J Work Environ Health 2010;36:404-12.

52. Larsen DL, Attkisson CC, Hargreaves WA, et al. Assessment of client/patient satisfaction: Development of a general scale. Eval Program Plann 1979;2:197-207.

53. Schmidt J, Nübling R. ZUF-8. Fragebogen zur messung der patientenzufriedenheit. In: Brähler E, Schumacher J, Strauß B. Hrsg Diagnostische verfahren in der psychotherapie. Göttingen: Hogrefe, 2002:392-6.

54. Rozental A, Boettcher J, Andersson G, et al. Negative effects of internet interventions: a qualitative content analysis of patients' experiences with treatments delivered online. Cogn Behav Ther 2015;44:223-36.

55. Wagner B, Riggs P, Mikulich-Gilbertson S. The importance of distribution-choice in modeling substance use data: a comparison of negative binomial, beta binomial, and zero-inflated distributions. Am J Drug Alcohol Abuse 2015;41:489-97.

56. Piaggio G, Elbourne DR, Pocock SJ, et al. Reporting of noninferiority and equivalence randomized trials: extension of the CONSORT 2010 statement. JAMA 2012;308:2594.

57. Wiens BL. Choosing an equivalence limit for noninferiority or equivalence studies. Control Clin Trials 2002;23:2-14.

58. Krauth $\mathrm{C}$, Hessel F, Hansmeier T, et al. [Empirical standard costs for health economic evaluation in Germany-a proposal by the working group methods in health economic evaluation]. Gesundheitswesen 2005;67:736-46.

59. Bock JO, Brettschneider $\mathrm{C}$, Seidl $\mathrm{H}$, et al. [Calculation of standardised unit costs from a societal perspective for health economic evaluation]. Gesundheitswesen 2015;77:53-61.

60. Bouwmans C, De Jong K, Timman R, et al. Feasibility, reliability and validity of a questionnaire on healthcare consumption and productivity loss in patients with a psychiatric disorder (TiC-P). BMC Health Serv Res 2013;13:217.

61. Herdman M, Gudex C, Lloyd A, et al. Development and preliminary testing of the new five-level version of EQ-5D (EQ-5D-5L). Qual Life Res 2011;20:1727-36.

62. Lamers LM, McDonnell J, Stalmeier PFM, et al. The Dutch tariff: results and arguments for an effective design for national EQ-5D valuation studies. Health Econ 2006;15:1121-32.

63. Greiner W, Claes C, Busschbach JJV, et al. Validating the EQ-5D with time trade off for the German population. Eur $J$ Health Econ 2005;6:124-30.

64. van Hout B, Janssen MF, et al. Interim scoring for the EQ-5D-5L Mapping the EQ-5D-5L to EQ-5D-3L value sets. Value in Health 2012;15:708-15.

65. van Hout $B$, Janssen MF, Feng YS, et al. Interim scoring for the EQ-5D-5L: mapping the EQ-5D-5L to EQ-5D-3L value sets. Value Health 2012;15:708-15.

66. Husereau D, Drummond M, Petrou S, et al. Consolidated Health Economic Evaluation Reporting Standards (CHEERS) statement. BMJ 2013;346:f1049-9.

67. Ramsey SD, Willke RJ, Glick $\mathrm{H}$, et al. Cost-effectiveness analysis alongside clinical trials II-an ISPOR Good Research Practices Task Force Report. Value Health 2015;18:161-72.

68. Drummond M, Sculpher M, Torrance G, et al. Methods for the economic evaluation of health care programmes. Oxford: Oxford University Press, 2005.

69. Hoch JS, Rockx MA, Krahn AD. Using the net benefit regression framework to construct cost-effectiveness acceptability curves: an example using data from a trial of external loop recorders versus Holter monitoring for ambulatory monitoring of "community acquired" syncope. BMC Health Serv Res 2006;6:68-107.

70. Isaranuwatchai W, Markle-Reid M, Hoch JS. Adjusting for baseline covariates in net benefit regression: how you adjust matters. Pharmacoeconomics 2015;33:1083-90.

71. Baker AL, Thornton LK, Hiles S, et al. Psychological interventions for alcohol misuse among people with co-occurring depression or anxiety disorders: a systematic review. J Affect Disord 2012:139:217-29. 